Revista de Matemática: Teoría y Aplicaciones 2001 8(1) : 1-12

CIMPA - UCR - CCSS ISSN: 1409-2433

\title{
DETECTING CONSTRAINT REDUNDANCY IN 0-1 LINEAR PROGRAMMING PROBLEMS
}

\author{
SusAnA MuÑOZ*
}

Received: 23 Octubre 2000

\begin{abstract}
Resumen
En este trabajo se presenta un procedimiento de obtención de cotas superiores para una función lineal a partir de ciertas familias de empaquetamientos, cubrimientos y conjuntos ordenados especiales. Asimismo, se presenta un nuevo método de detección de restricciones redundantes en problemas de programación lineal 0-1 basado en dichas cotas que permite considerar conjuntamente varias restricciones. Además, se muestra una situación de redundancia que es detectada por este método, pero no por los métodos tradicionales, los cuales consideran las restricciones individualmente.
\end{abstract}

Palabras clave: Restricciones redundantes, empaquetamientos, cubrimientos, conjuntos ordenados especiales, familias admisibles

\begin{abstract}
In this paper we present a procedure for obtaining upper bounds on a linear function by means of certain families of packings, coverings and special ordered sets. We also present a new method for detecting redundant constraints in 0-1 linear programming problems based on these bounds that allows consideration of several constraints jointly. Furthermore, we show a redundancy situation which is detected by this new method, but not by the traditional methods, which consider the constraints individually.
\end{abstract}

Keywords: Redundant constraints, packings, coverings, special ordered sets, admissible families

Mathematics Subject Classification: 90C10,90C05

\footnotetext{
*Departamento de Estadística e Investigación Operativa I, Facultad de Ciencias Matemáticas, Universidad Complutense de Madrid, Ciudad Universitaria, 28040 Madrid, Spain. E-mail: smunoz@eucmax.sim.ucm.es
} 


\section{Introduction}

Consider the 0-1 linear programming problem

$$
\max \left\{\sum_{j \in J} c_{j} x_{j} \mid \sum_{j \in J} a_{i j} x_{j} \sim b_{i} \quad \forall i \in I, \quad x_{j} \in\{0,1\} \quad \forall j \in J\right\},
$$

where $J=\{1, \ldots, n\}, I=\{1, \ldots, m\},\left\{c_{j}\right\}_{j \in J},\left\{a_{i j}\right\}_{i \in I, j \in J},\left\{b_{i}\right\}_{i \in I}$ are rational numbers and $\sim$ is the sense of each constraint $(\leq, \geq,=)$.

In integer programming there are many ways of representing the same problem, and the choice of formulation is of crucial importance to solving it (see e.g. [8, 10, 13, 14]). Preprocessing attempts to improve the initial formulation by using several automatic techniques such as infeasibility and redundancy detection, variable fixing and constraint reformulation (see $[3,8,9,12,14]$ among many others). It is well known that preprocessing techniques can considerably reduce the time required to solve large-scale integer programming problems.

The $L P$ relaxation of $(P)$ is the same problem $(P)$ where each variable $x_{j}$ is allowed to take any value in the interval $[0,1]$.

Detection of redundant constraints in $(P)$ (i.e., constraints whose elimination does not add any feasible solution to the LP relaxation of $(P))$ is based on computing bounds on a linear function $z$ whose variables $\left(x_{j}\right)_{j \in J}$ are restricted to take values in a certain subset of $[0,1]^{n}$. Obviously, the best bound is the one given by the optimal value of $z$ in the associated optimization problem. However, in general, this problem is not easy to solve, since it is similar to the LP relaxation of $(P)$, see Section 4. Hence, there is a need to develop simple procedures for obtaining bounds on $z$.

In easy terms, a packing, a covering and a special ordered set can be considered as subsets of indices of $0-1$ variables where at most, at least and exactly one such variable, respectively, can take the value 1 . These structures may appear explicitly in the problem, but can also be derived from the constraint system by using probing techniques (see e.g. $[1,7,14]$ ). Other methods for packing identification can be found in $[4,12]$ (see also [11]).

The earliest papers dealing with obtaining bounds on linear functions consider only the coefficients of those functions (see [3, 14] among others). In 1985, Johnson, Kostreva and Suhl introduced a more advanced procedure that makes use of information from families of pairwise disjoint packings (see [8,9]) and, in 1996, Escudero, Garín and Pérez improved this procedure allowing overlapping among certain pairs of packings (see [5, 12]).

This is a theoretical paper whose contribution is twofold. First, we extend the procedure given in [5] to obtain upper bounds on linear functions, using certain families of packings, coverings and special ordered sets, so-called admissible families. Secondly, we present a new method for detecting constraint redundancy in 0-1 linear programming problems that allows consideration of several constraints jointly. This method can easily be generalized to mixed integer programming problems with bounded variables (see e.g. [14]). 
The paper is organized as follows: Section 2 reviews the concepts of packings, coverings and special ordered sets. Section 3 introduces the concept of admissible families, describes a procedure for obtaining upper bounds on a linear function based on this type of families, and provides an example in which the procedures using only families of packings obtain worse upper bounds. Section 4 presents our method for detecting redundant constraints in problem $(P)$. It also shows a situation detected by this method, but not by the methods available in current literature, which consider single constraints. Finally, Section 5 draws some conclusions from this work.

\section{Packings, coverings and special ordered sets}

Given a set of variables $\left\{x_{1}, \ldots, x_{n}\right\}$ and a set $F \subseteq\{1, \ldots, n\}$, let $X(F)$ denote the sum of the variables whose indices belong to $F$, that is, $X(F)=\sum_{j \in F} x_{j}$.

Based on the notation used in [2], we define the following concepts:

Definition 1. A packing $C$ is a non-empty subset of indices of 0-1 variables that induces the constraint $X\left(C^{+}\right)-X\left(C^{-}\right) \leq 1-\left|C^{-}\right|$, where $C^{+} \cup C^{-}=C$ and $C^{+} \cap C^{-}=\emptyset$.

Definition 2. A covering $C$ is a non-empty subset of indices of 0-1 variables that induces the constraint $X\left(C^{+}\right)-X\left(C^{-}\right) \geq 1-\left|C^{-}\right|$, where $C^{+} \cup C^{-}=C$ and $C^{+} \cap C^{-}=\emptyset$.

Definition 3. A special ordered set $C$ is a non-empty subset of indices of 0-1 variables that induces the constraint $X\left(C^{+}\right)-X\left(C^{-}\right)=1-\left|C^{-}\right|$, where $C^{+} \cup C^{-}=C$ and $C^{+} \cap C^{-}=$ $\emptyset$.

Lemma 1 proves that any proper subset of a packing or of a special ordered set is a packing.

Lemma 1. Let $C$ be a packing or a special ordered set, let $C^{\prime}$ be a proper subset of $C$ and let $\left(x_{j}\right)_{j \in C} \in\{0,1\}^{|C|}$ be a feasible solution for the constraint induced by $C$. Then $\sum_{j \in C^{\prime} \cap C^{+}} x_{j}-\sum_{j \in C^{\prime} \cap C^{-}} x_{j} \leq 1-\left|C^{\prime} \cap C^{-}\right|$.

Proof. Since $C^{\prime} \cap C^{+}=C^{+} \backslash\left(C^{+} \backslash C^{\prime}\right), C^{\prime} \cap C^{-}=C^{-} \backslash\left(C^{-} \backslash C^{\prime}\right), \sum_{j \in C^{+}} x_{j}-\sum_{j \in C^{-}} x_{j} \leq$ $1-\left|C^{-}\right|$and $x_{j} \in\{0,1\} \quad \forall j \in C$, we obtain that $\sum_{j \in C^{\prime} \cap C^{+}} x_{j}-\sum_{j \in C^{\prime} \cap C^{-}} x_{j}=\sum_{j \in C^{+}} x_{j}-$ $\sum_{j \in C^{-}} x_{j}-\sum_{j \in C^{+} \backslash C^{\prime}} x_{j}+\sum_{j \in C^{-} \backslash C^{\prime}} x_{j} \leq 1-\left|C^{-}\right|+\left|C^{-} \backslash C^{\prime}\right|=1-\left|C^{\prime} \cap C^{-}\right|$. 


\section{Obtaining upper bounds on a linear function}

Definition 4. A constraint with variables $x_{1}, \ldots, x_{n}$ is said to be valid for a set $R \subseteq \mathbb{R}^{n}$ if it is satisfied by any vector $\left(x_{1}, \ldots, x_{n}\right) \in R$.

Definition 5. Let $\left\{C_{k}\right\}_{k \in K_{1}},\left\{C_{k}\right\}_{k \in K_{2}}$ and $\left\{C_{k}\right\}_{k \in K_{3}}$ be a family of packings, coverings and special ordered sets respectively. The family $\left\{C_{k}\right\}_{k \in K}$, where $K=K_{1} \cup K_{2} \cup K_{3}$, is said to be admissible for a set $R \subseteq\{0,1\}^{n}$ if the constraints induced by $\left\{C_{k}\right\}_{k \in K}$ are valid for $R$ and each set $K_{l}$ with $l \in\{1,2,3\}$ can be expressed as the union of three pairwise disjoint sets, say $D_{l}, S_{l}$ and $\bar{S}_{l}$, that satisfy the following conditions, where $D=D_{1} \cup D_{2} \cup D_{3}$, $S=S_{1} \cup S_{2} \cup S_{3}$ and $\bar{S}=\bar{S}_{1} \cup \bar{S}_{2} \cup \bar{S}_{3}$ :

(1) If $k \in D$ and $k^{\prime} \in K \backslash\{k\}$, then $C_{k} \cap C_{k^{\prime}}=\emptyset$.

(2) For each $k \in S$ there exists a unique $\bar{s}(k) \in \bar{S}$ such that $C_{k} \cap C_{\bar{s}(k)}=\left(C_{k}^{+} \cap C_{\bar{s}(k)}^{+}\right) \cup$ $\left(C_{k}^{-} \cap C_{\bar{s}(k)}^{-}\right) \neq \emptyset$, and $C_{k} \cap C_{k^{\prime}}=\emptyset \quad \forall k^{\prime} \in(S \backslash\{k\}) \cup(\bar{S} \backslash\{\bar{s}(k)\})$.

(3) For each $k \in \bar{S}$ there exists a unique $s(k) \in S$ such that $\bar{s}(s(k))=k$, and $C_{k} \cap C_{k^{\prime}}=\emptyset$ $\forall k^{\prime} \in \bar{S} \backslash\{k\}$.

Note. By conditions (2) and (3) above, we have that $|S|=|\bar{S}|$.

All of the results stated from now on can be generalized to admissible families such that the constraints induced by $\left\{C_{k}\right\}_{k \in K}$ are of the form $X\left(C_{k}^{+}\right)-X\left(C_{k}^{-}\right) \leq n_{k}-\left|C_{k}^{-}\right|$, $X\left(C_{k}^{+}\right)-X\left(C_{k}^{-}\right) \geq n_{k}-\left|C_{k}^{-}\right|$and $X\left(C_{k}^{+}\right)-X\left(C_{k}^{-}\right)=n_{k}-\left|C_{k}^{-}\right|$, where $n_{k}$ is an integer with $1 \leq n_{k} \leq\left|C_{k}\right|$.

Given a non-empty set $R \subseteq\{0,1\}^{n}$, we are interested in obtaining upper bounds on a function $z=\sum_{j \in J} a_{j} x_{j}$, where $\left\{a_{j}\right\}_{j \in J}$ are rationals and $\left(x_{j}\right)_{j \in J} \in R$. For that, we consider an admissible family for $R$, say $\mathcal{C}=\left\{C_{k}\right\}_{k \in K}$, where $K=K_{1} \cup K_{2} \cup K_{3}$ and $\left\{C_{k}\right\}_{k \in K_{1}},\left\{C_{k}\right\}_{k \in K_{2}}$ and $\left\{C_{k}\right\}_{k \in K_{3}}$ are a family of packings, coverings and special ordered sets respectively. Without loss of generality let us assume that $C_{k}^{-}=\emptyset$ for each $k \in K$ (otherwise, it suffices to substitute $x_{j}$ by $1-x_{j}^{\prime} \quad \forall j \in \bigcup_{k \in K} C_{k}^{-}$).

Let $u_{z, \mathcal{C}}=\max \left\{\sum_{j \in J} a_{j} x_{j} \mid\left(x_{j}\right)_{j \in J} \in R_{\mathcal{C}}\right\}$, where $R_{\mathcal{C}}=\left\{\left(x_{j}\right)_{j \in J} \in[0,1]^{n} \mid X\left(C_{k}\right) \leq 1\right.$ $\left.\forall k \in K_{1}, X\left(C_{k}\right) \geq 1 \quad \forall k \in K_{2}, X\left(C_{k}\right)=1 \quad \forall k \in K_{3}\right\}$ (if $K=\emptyset$, we define $R_{\mathcal{C}}=[0,1]^{n}$ ). Then $R \subseteq R_{\mathcal{C}}$, since the constraints induced by $\left\{C_{k}\right\}_{k \in K}$ are valid for $R$. Thus, $u_{z, \mathcal{C}}$ is an upper bound on the function $z$.

Below we give some cases where eliminating one of the elements of $\mathcal{C}$ leaves the set $R_{\mathcal{C}}$ unchanged and, so, the bound $u_{z, \mathcal{C}}$ also remains unchanged. 
Let $k, k^{\prime} \in S \cup \bar{S}$ be such that $C_{k} \subset C_{k^{\prime}}$.

- If $k \in S_{1} \cup \bar{S}_{1}$ and $k^{\prime} \in S_{1} \cup \bar{S}_{1} \cup S_{3} \cup \bar{S}_{3}$, we can eliminate $k$ from $S_{1} \cup \bar{S}_{1}$ and move $k^{\prime}$ to $D_{1} \cup D_{3}$.

- If $k \in S_{1} \cup \bar{S}_{1},\left|C_{k}\right|=1$ and $k^{\prime} \in S_{2} \cup \bar{S}_{2}$, we can eliminate $k$ from $S_{1} \cup \bar{S}_{1}$ and move $k^{\prime}$ to $D_{2}$.

- If $k \in S_{2} \cup \bar{S}_{2} \cup S_{3} \cup \bar{S}_{3}$ and $k^{\prime} \in S_{2} \cup \bar{S}_{2}$, we can eliminate $k^{\prime}$ from $S_{2} \cup \bar{S}_{2}$ and move $k$ to $D_{2} \cup D_{3}$.

- If $k \in S_{2} \cup \bar{S}_{2}$ and $k^{\prime} \in S_{1} \cup \bar{S}_{1} \cup S_{3} \cup \bar{S}_{3}$, we can fix $x_{j}=0 \quad \forall j \in C_{k^{\prime}} \backslash C_{k}$, eliminate $k^{\prime}$ from $S_{1} \cup \bar{S}_{1} \cup S_{3} \cup \bar{S}_{3}$ and move $k$ to $D_{3}$.

- If $k \in S_{3} \cup \bar{S}_{3}$ and $k^{\prime} \in S_{1} \cup \bar{S}_{1} \cup S_{3} \cup \bar{S}_{3}$, we can fix $x_{j}=0 \quad \forall j \in C_{k^{\prime}} \backslash C_{k}$, eliminate $k^{\prime}$ from $S_{1} \cup \bar{S}_{1} \cup S_{3} \cup \bar{S}_{3}$ and move $k$ to $D_{3}$.

Now, let $k \in D$ be such that $C_{k}=\{j\}$.

- If $k \in D_{1}$, we can eliminate $k$ from $D_{1}$.

- If $k \in D_{2} \cup D_{3}$, we can fix $x_{j}=1$ and eliminate $k$ from $D_{2} \cup D_{3}$.

Therefore, we can assume that, for each $k \in K,\left|C_{k}\right|>1$ and, if $\exists k^{\prime} \in K \backslash\{k\}$ such that $C_{k} \subset C_{k^{\prime}}$, then $k \in S_{1} \cup \bar{S}_{1}$ and $k^{\prime} \in S_{2} \cup \bar{S}_{2}$.

Any non-empty subset of $R_{\mathcal{C}}$ verifies that, if $\left(x_{j}\right)_{j \in J}$ is restricted to belong to that subset, then $u_{z, \mathcal{C}}$ is still an upper bound on $z$. Consequently, whenever $u_{z, \mathcal{C}}$ is mentioned, it will be assumed that $\left(x_{j}\right)_{j \in J}$ can take any value in $R_{\mathcal{C}}$ and the initial set $R$ will be allowed to be empty. (Note that $R_{\mathcal{C}} \neq \emptyset$ ).

Lemma 2. Let $R_{z, b}=\left\{\left(x_{j}\right)_{j \in J} \in[0,1]^{n} \mid \sum_{j \in J} a_{j} x_{j} \leq b\right\}$, where $b$ is a rational constant. Then $R_{\mathcal{C}} \subseteq R_{z, b}$ if and only if $u_{z, \mathcal{C}} \leq b$.

Proof. It follows from the definition of $u_{z, \mathcal{C}}$.

Given a set $C \subseteq J$, we define $\bar{\varphi}(C)=\max \left\{a_{j} \mid j \in C\right\}$ and $\bar{\varphi}_{0}(C)=\max \{\bar{\varphi}(C), 0\}$. Let $J^{+}=\left\{j \in J \mid a_{j}>0\right\}, J^{-}=\left\{j \in J \mid a_{j}<0\right\}, J^{0}=\left\{j \in J \mid a_{j}=0\right\}, T=\bigcup_{k \in K} C_{k}$ and 


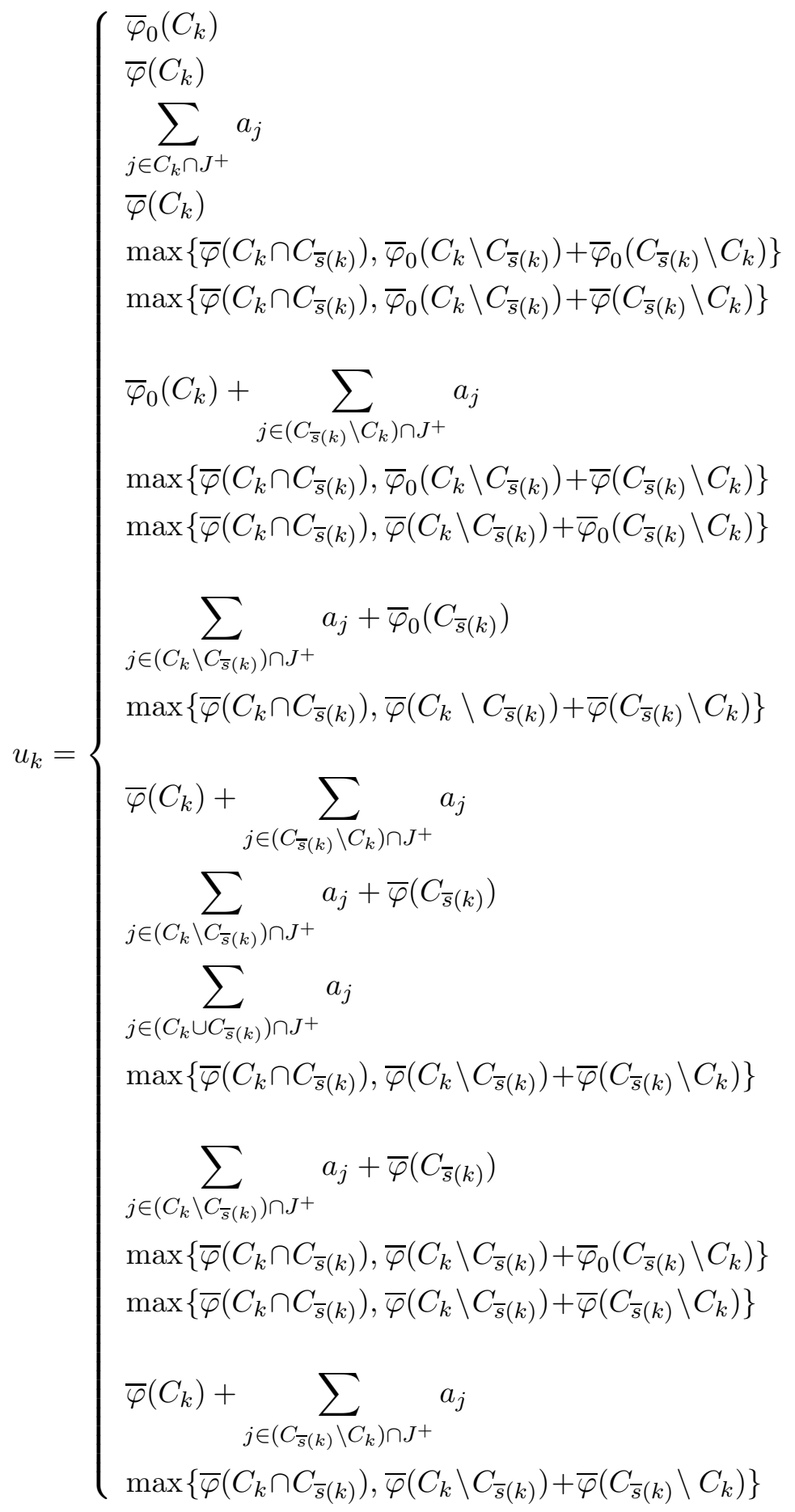

$\forall k \in D_{1}$

$\forall k \in D_{2}$ such that $C_{k} \subseteq J^{-}$

$\forall k \in D_{2}$ such that $C_{k} \nsubseteq J^{-}$

$\forall k \in D_{3}$

$\forall k \in S_{1}$ such that $\bar{s}(k) \in \bar{S}_{1}$

$\forall k \in S_{1}$ such that $\bar{s}(k) \in \bar{S}_{2}$

and $C_{\bar{s}(k)} \backslash C_{k} \subseteq J^{-}$

$\forall k \in S_{1}$ such that $\bar{s}(k) \in \bar{S}_{2}$ and $C_{\bar{s}(k)} \backslash C_{k} \nsubseteq J^{-}$

$\forall k \in S_{1}$ such that $\bar{s}(k) \in \bar{S}_{3}$

$\forall k \in S_{2}$ such that $\bar{s}(k) \in \bar{S}_{1}$

and $C_{k} \backslash C_{\bar{s}(k)} \subseteq J^{-}$

$\forall k \in S_{2}$ such that $\bar{s}(k) \in \bar{S}_{1}$ and $C_{k} \backslash C_{\bar{s}(k)} \nsubseteq J^{-}$

$\forall k \in S_{2}$ such that $\bar{s}(k) \in \bar{S}_{2}$ and $C_{k} \cup C_{\bar{s}(k)} \subseteq J^{-}$

$\forall k \in S_{2}$ such that $\bar{s}(k) \in \bar{S}_{2}$, $C_{k} \subseteq J^{-}$and $C_{\bar{s}(k)} \backslash C_{k} \nsubseteq J^{-}$ $\forall k \in S_{2}$ such that $\bar{s}(k) \in \bar{S}_{2}$, $C_{k} \backslash C_{\bar{s}(k)} \not J^{-}$and $C_{\bar{s}(k)} \subseteq J^{-}$ $\forall k \in S_{2}$ such that $\bar{s}(k) \in \bar{S}_{2}$, $C_{k} \nsubseteq J^{-}$and $C_{\bar{s}(k)} \nsubseteq J^{-}$ $\forall k \in S_{2}$ such that $\bar{s}(k) \in \bar{S}_{3}$ and $C_{k} \backslash C_{\bar{s}(k)} \subseteq J^{-}$

$\forall k \in S_{2}$ such that $\bar{s}(k) \in \bar{S}_{3}$ and $C_{k} \backslash C_{\bar{s}(k)} \nsubseteq J^{-}$

$\forall k \in S_{3}$ such that $\bar{s}(k) \in \bar{S}_{1}$ $\forall k \in S_{3}$ such that $\bar{s}(k) \in \bar{S}_{2}$ and $C_{\bar{s}(k)} \backslash C_{k} \subseteq J^{-}$ $\forall k \in S_{3}$ such that $\bar{s}(k) \in \bar{S}_{2}$ and $C_{\bar{s}(k)} \backslash C_{k} \nsubseteq J^{-}$ $\forall k \in S_{3}$ such that $\bar{s}(k) \in \bar{S}_{3}$

Theorem 1. $u_{z, \mathcal{C}}=\sum_{k \in D \cup S} u_{k}+\sum_{j \in J^{+} \backslash T} a_{j}$.

Proof. Since the sets $\left\{C_{k}\right\}_{k \in D},\left\{C_{k} \cup C_{\bar{s}(k)}\right\}_{k \in S}$ are pairwise disjoint, it can easily be 
verified that $\sum_{j \in J} a_{j} x_{j} \leq \sum_{k \in D \cup S} u_{k}+\sum_{j \in J^{+} \backslash T} a_{j} \forall\left(x_{j}\right)_{j \in J} \in R_{\mathcal{C}}$. On the other hand, it is clear that $\exists\left(x_{j}^{*}\right)_{j \in J} \in R_{\mathcal{C}}$ such that $\sum_{j \in C_{k}} a_{j} x_{j}^{*}=u_{k} \quad \forall k \in D, \sum_{j \in C_{k} \cup C_{\bar{s}(k)}} a_{j} x_{j}^{*}=u_{k} \quad \forall k \in S$ and $\sum_{j \in J \backslash T} a_{j} x_{j}^{*}=\sum_{j \in J^{+} \backslash T} a_{j}$. Hence, we have that $\sum_{j \in J} a_{j} x_{j}^{*}=\sum_{k \in D \cup S} u_{k}+\sum_{j \in J^{+} \backslash T} a_{j}$, which proves the assertion.

Corollary 1. Let $z^{\prime}=\lambda z$, where $\lambda$ is a non-negative rational constant. Then $u_{z^{\prime}, \mathcal{C}}=$ $\lambda u_{z, \mathcal{C}}$.

Corollary 2. $u_{z, \mathcal{C}} \geq 0$ if $K_{2}=K_{3}=\emptyset$.

In general, there will exist several admissible families for the set $R$. Example 1 illustrates the great variations in the value that $u_{z, \mathcal{C}}$ takes depending on the family $\mathcal{C}$ that has been selected; this demonstrates the importance of making a good choice.

EXAmPle 1. Let $z=x_{1}-4 x_{2}+2 x_{3}+6 x_{4}-x_{5}-3 x_{6}-5 x_{7}+4 x_{8}$ and let $R$ be the set of solutions $\left(x_{1}, \ldots, x_{8}\right) \in\{0,1\}^{8}$ that satisfy the following constraints:

$$
\begin{array}{rlrl}
x_{1}+x_{2}+x_{3} & +x_{7} & \leq 1 \\
x_{3}+x_{4} & +x_{7} & \leq 1 \\
x_{2} & & & \leq x_{6}+x_{7} \\
& & \geq 1 \\
x_{4}+x_{5}+x_{8} & =1
\end{array}
$$

Consider the packings $C_{1}=\{1,2,3,7\}$ and $C_{2}=\{3,4,7\}$, the covering $C_{3}=\{2,6,7\}$ and the special ordered set $C_{4}=\{4,5,8\}$. (Note that constraints (1)-(4) are induced by $C_{1}, \ldots, C_{4}$ respectively).

The family $\left\{C_{1}, C_{2}, C_{3}, C_{4}\right\}$ is not admissible for $R$, since $C_{1} \cap C_{2} \cap C_{3} \neq \emptyset$. Nevertheless, by Lemma 1 any non-empty set $C \subset C_{k}$, where $k \in\{1,2,4\}$, is a packing whose induced constraint $X(C) \leq 1$ is valid for $R$.

Let $\mathcal{C}^{\prime}=\left\{C_{1}^{\prime}, C_{2}^{\prime}, C_{3}^{\prime}\right\}$, where $C_{1}^{\prime}=\{1,2,3,7\}, C_{2}^{\prime}=\{3,4,7\}$ and $C_{3}^{\prime}=\{5,8\}$. Taking $D_{1}=\{3\}, S_{1}=\{1\}, \bar{S}_{1}=\{2\}$ and $D_{2}=S_{2}=\bar{S}_{2}=D_{3}=S_{3}=\bar{S}_{3}=\emptyset$ we have that $\mathcal{C}^{\prime}$ is an admissible family for $R$ and, by Theorem $1, u_{z, \mathcal{C}^{\prime}}=u_{1}+u_{3}=\max \{2,1+6\}+4=11$.

Let $\mathcal{C}^{\prime \prime}=\left\{C_{1}^{\prime \prime}, C_{2}^{\prime \prime}, C_{3}^{\prime \prime}\right\}$, where $C_{1}^{\prime \prime}=\{3,4,7\}, C_{2}^{\prime \prime}=\{2,6,7\}$ and $C_{3}^{\prime \prime}=\{5,8\}$. Taking $D_{1}=\{3\}, S_{1}=\{1\}, \bar{S}_{2}=\{2\}$ and $\bar{S}_{1}=D_{2}=S_{2}=D_{3}=S_{3}=\bar{S}_{3}=\emptyset$ we have that $\mathcal{C}^{\prime \prime}$ is an admissible family for $R$ and, by Theorem $1, u_{z, \mathcal{C}^{\prime \prime}}=u_{1}+u_{3}+a_{1}=\max \{-5,6-3\}+$ $4+1=8$.

Let $\mathcal{C}^{\prime \prime \prime}=\left\{C_{1}^{\prime \prime \prime}, C_{2}^{\prime \prime \prime}, C_{3}^{\prime \prime \prime}, C_{4}^{\prime \prime \prime}\right\}$, where $C_{1}^{\prime \prime \prime}=\{1,2,7\}, C_{2}^{\prime \prime \prime}=\{3,4\}, C_{3}^{\prime \prime \prime}=\{2,6,7\}$ and $C_{4}^{\prime \prime \prime}=\{4,5,8\}$. Taking $S_{1}=\{1,2\}, \bar{S}_{2}=\{3\}, \bar{S}_{3}=\{4\}$ and $D_{1}=\bar{S}_{1}=D_{2}=$ $S_{2}=D_{3}=S_{3}=\emptyset$ we have that $\mathcal{C}^{\prime \prime \prime}$ is an admissible family for $R$ and, by Theorem 1 , $u_{z, \mathcal{C}^{\prime \prime \prime}}=u_{1}+u_{2}=\max \{-4,1-3\}+\max \{6,2+4\}=4$. 
The best upper bound on the function $z$ is given by $\mathcal{C}^{\prime \prime \prime}$, since $u_{z, \mathcal{C}^{\prime}}>u_{z, \mathcal{C}^{\prime \prime}}>u_{z, \mathcal{C}^{\prime \prime \prime}}$. Furthermore, choosing $x_{1}=x_{4}=x_{6}=1$ and $x_{2}=x_{3}=x_{5}=x_{7}=x_{8}=0$, we obtain that $\left(x_{1}, \ldots, x_{8}\right) \in R$ and $x_{1}-4 x_{2}+2 x_{3}+6 x_{4}-x_{5}-3 x_{6}-5 x_{7}+4 x_{8}=4$. Consequently, there is no upper bound on $z$ stronger than $u_{z, \mathcal{C}^{\prime \prime \prime}}$. (Note that if one restricts the admissible families for $R$ to families of packings, as the traditional procedures do, then the associated upper bounds on $z$ will be greater than 4 , since $\max \left\{x_{1}-4 x_{2}+2 x_{3}+6 x_{4}-x_{5}-3 x_{6}-5 x_{7}+4 x_{8} \mid\right.$ $\left.\left.\sum_{j \in C_{k}} x_{j} \leq 1 \quad \forall k \in\{1,2,4\}, x_{j} \in[0,1] \quad \forall j \in\{1, \ldots, 8\}\right\}=7\right)$.

\section{Detecting constraint redundancy}

Let $r \in I, R_{r}^{-}=\left\{\left(x_{j}\right)_{j \in J} \in[0,1]^{n} \mid \sum_{j \in J} a_{i j} x_{j} \sim b_{i} \quad \forall i \in I \backslash\{r\}\right\}$ and $R_{r}=\left\{\left(x_{j}\right)_{j \in J} \in\right.$ $\left.[0,1]^{n} \mid \sum_{j \in J} a_{r j} x_{j} \sim b_{r}\right\}$. We are interested in determining whether the $r$ th constraint is redundant, that is, whether $R_{r}^{-} \subseteq R_{r}$ (in this case the $r$ th constraint can be dropped from $(P)$, although it may be preferable not to do so, since, in some cases, redundant constraints can be converted into non-redundant ones by applying coefficient increasing or reduction methods, see [6]).

Let $R=\left\{\left(x_{j}\right)_{j \in J} \in\{0,1\}^{n} \mid \sum_{j \in J} a_{i j} x_{j} \sim b_{i} \quad \forall i \in I\right\}$ and let $\mathcal{C}=\left\{C_{k}\right\}_{k \in K}$ be an admissible family for $R$, where $K=K_{1} \cup K_{2} \cup K_{3}$ and $\left\{C_{k}\right\}_{k \in K_{1}},\left\{C_{k}\right\}_{k \in K_{2}}$ and $\left\{C_{k}\right\}_{k \in K_{3}}$ are a family of packings, coverings and special ordered sets respectively. The family $\mathcal{C}$ will be obtained as follows:

We start by identifying a family $\mathcal{C}_{0}$ of packings, coverings and special ordered sets whose induced constraints are valid for $R$ and that contains the family $\mathcal{C}_{P}$ of packings, coverings and special ordered sets that induce contraints of problem $(P)$, see Section 1 . If $\mathcal{C}_{0}$ is an admissible family for $R$, we take $\mathcal{C}=\mathcal{C}_{0}$; otherwise, by Lemma 1 it is easy to determine an admissible family for $R$ from $\mathcal{C}_{0}$, see Example 1 . (For simplicity, we can assume that, for each $k \in K, C_{k}^{-}=\emptyset,\left|C_{k}\right|>1$ and, if $\exists k^{\prime} \in K \backslash\{k\}$ such that $C_{k} \subset C_{k^{\prime}}$, then $k \in S_{1} \cup \bar{S}_{1}$ and $k^{\prime} \in S_{2} \cup \bar{S}_{2}$ ).

Let $I_{\mathcal{C}}$ be the set of indices of the constraints in $(P)$ that are induced by $\left\{C_{k}\right\}_{k \in K}$.

Without loss of generality, from now on we assume that every constraint in $(P)$ is an inequality of type $\leq$. (Note that any inequality of type $\geq$ can be converted into another one of type $\leq$ by multiplying it by -1 , and any equality can be decomposed into two inequalities).

Proposition 1. Let $i_{1}, \ldots, i_{p} \in I \backslash I_{\mathcal{C}}$ be such that $i_{l} \neq i_{l^{\prime}} \quad \forall l, l^{\prime} \in\{1, \ldots, p\}$ with $l \neq l^{\prime}$, and let $z=\sum_{j \in J}\left(\lambda_{1} a_{i_{1} j}-\lambda_{2} a_{i_{2} j}-\ldots-\lambda_{p} a_{i_{p} j}\right) x_{j}$, where $p \geq 1$ and $\lambda_{1}, \ldots, \lambda_{p}$ are positive integers relatively prime. If $u_{z, \mathcal{C}} \leq \lambda_{1} b_{i_{1}}-\lambda_{2} b_{i_{2}}-\ldots-\lambda_{p} b_{i_{p}}$ and $R_{i_{1}}^{-} \subseteq R_{\mathcal{C}}$, then the $i_{1}$ th constraint is redundant. 
PROOF. Let $b=\lambda_{1} b_{i_{1}}-\lambda_{2} b_{i_{2}}-\ldots-\lambda_{p} b_{i_{p}}$. If $u_{z, \mathcal{C}} \leq b$ and $R_{i_{1}}^{-} \subseteq R_{\mathcal{C}}$, by Lemma 2 it follows that $R_{i_{1}}^{-} \subseteq R_{z, b}$. Moreover, for each $l \in\{2, \ldots, p\}$ we have that $\sum_{j \in J} a_{i_{l} j} x_{j} \leq b_{i_{l}}$ $\forall\left(x_{j}\right)_{j \in J} \in R_{i_{1}}^{-}$, since $i_{1} \neq i_{l}$. Thus, $\lambda_{1} \sum_{j \in J} a_{i_{1} j} x_{j} \leq \lambda_{1} b_{i_{1}}+\sum_{j \in J}\left(\lambda_{2} a_{i_{2} j}+\ldots+\lambda_{p} a_{i_{p} j}\right) x_{j}-$ $\lambda_{2} b_{i_{2}}-\ldots-\lambda_{p} b_{i_{p}} \leq \lambda_{1} b_{i_{1}} \quad \forall\left(x_{j}\right)_{j \in J} \in R_{i_{1}}^{-}$, hence $R_{i_{1}}^{-} \subseteq R_{i_{1}}$.

In Proposition 1 it is not necessary to impose the condition that $\lambda_{1}, \ldots, \lambda_{p}$ be integers relatively prime. Assuming they are integers, $\lambda_{1} b_{i_{1}}-\lambda_{2} b_{i_{2}}-\ldots-\lambda_{p} b_{i_{p}}$ and the coefficients of the function $z$ are rationals. On the other hand, if $\lambda_{1}, \ldots, \lambda_{p}$ are positive integers, it follows from Corollary 1 that the result of applying Proposition 1 by considering $\lambda_{1}, \ldots, \lambda_{p}$ is the same as by considering $\frac{\lambda_{1}}{M}, \ldots, \frac{\lambda_{p}}{M}$, where $M$ is the greatest common divisor of $\lambda_{1}, \ldots, \lambda_{p}$. Therefore, in order to make the calculation of $\lambda_{1} b_{i_{1}}-\lambda_{2} b_{i_{2}}-\ldots-\lambda_{p} b_{i_{p}}$ and $u_{z, \mathcal{C}}$ easier, it is advisable that $\lambda_{1}, \ldots, \lambda_{p}$ be relatively prime (if $p=1$, we will take $\lambda_{1}=1$ ).

If when applying Proposition 1 one obtains that $u_{z, \mathcal{C}} \leq \lambda_{1} b_{i_{1}}-\lambda_{2} b_{i_{2}}-\ldots-\lambda_{p} b_{i_{p}}$ and $R_{i_{1}}^{-} \not R_{\mathcal{C}}$, then adding the constraints induced by $\left\{C_{k}\right\}_{k \in K\left(i_{1}\right)}$ to the constraint system of $(P)$, where $K\left(i_{1}\right)=\left\{k \in K_{1} \mid R_{i_{1}}^{-} \nsubseteq\left\{\left(x_{j}\right)_{j \in J} \in[0,1]^{n} \mid X\left(C_{k}\right) \leq 1\right\}\right\} \cup\left\{k \in K_{2} \mid R_{i_{1}}^{-} \nsubseteq\right.$ $\left.\left\{\left(x_{j}\right)_{j \in J} \in[0,1]^{n} \mid X\left(C_{k}\right) \geq 1\right\}\right\} \cup\left\{k \in K_{3} \mid R_{i_{1}}^{-} \nsubseteq\left\{\left(x_{j}\right)_{j \in J} \in[0,1]^{n} \mid X\left(C_{k}\right)=1\right\}\right\}$, we have that $R_{i_{1}}^{-} \subseteq R_{\mathcal{C}}$ and, consequently, the $i_{1}$ th constraint is redundant.

It is clear that Proposition 1 also holds for any indices $i_{2}, \ldots, i_{p} \in I$ such that $i_{1} \neq i_{l} \quad \forall l \in\{2, \ldots p\}$. Now, Lemma 3 proves that if some of them belong to $I_{\mathcal{C}}$ and Proposition 1 detects the redundancy of the $i_{1}$ th constraint by considering $i_{2}, \ldots, i_{p}$, then it will also detect it by considering only those indices in $I \backslash I_{\mathcal{C}}$.

Lemma 3. Let $i_{1}, \ldots, i_{p^{\prime}} \in I \backslash I_{\mathcal{C}}$ be such that $i_{l} \neq i_{l^{\prime}} \quad \forall l, l^{\prime} \in\left\{1, \ldots, p^{\prime}\right\}$ with $l \neq l^{\prime}$, let $i_{p^{\prime}+1}, \ldots, i_{p} \in I_{\mathcal{C}}$ be such that $i_{l} \neq i_{l^{\prime}} \quad \forall l, l^{\prime} \in\left\{p^{\prime}+1, \ldots, p\right\}$ with $l \neq l^{\prime}$, let $z=$ $\sum_{j \in J}\left(\lambda_{1} a_{i_{1} j}-\lambda_{2} a_{i_{2} j}-\ldots-\lambda_{p} a_{i_{p} j}\right) x_{j}$ and $z^{\prime}=\sum_{j \in J}\left(\lambda_{1} a_{i_{1} j}-\lambda_{2} a_{i_{2} j}-\ldots-\lambda_{p^{\prime}} a_{i_{p^{\prime}} j}\right) x_{j}$, where $1 \leq p^{\prime}<p$ and $\lambda_{1}, \ldots, \lambda_{p}$ are positive integers relatively prime. If $u_{z, \mathcal{C}} \leq \lambda_{1} b_{i_{1}}-$ $\lambda_{2} b_{i_{2}}-\ldots-\lambda_{p} b_{i_{p}}$, then $u_{z^{\prime}, \mathcal{C}} \leq \lambda_{1} b_{i_{1}}-\lambda_{2} b_{i_{2}}-\ldots-\lambda_{p^{\prime}} b_{i_{p^{\prime}}}$.

ProOF. Let $b=\lambda_{1} b_{i_{1}}-\lambda_{2} b_{i_{2}}-\ldots-\lambda_{p} b_{i_{p}}$ and $b^{\prime}=\lambda_{1} b_{i_{1}}-\lambda_{2} b_{i_{2}}-\ldots-\lambda_{p^{\prime}} b_{i_{p^{\prime}}}$. If $u_{z, \mathcal{C}} \leq b$, by Lemma 2 it follows that $R_{\mathcal{C}} \subseteq R_{z, b}$ and, since $i_{p^{\prime}+1}, \ldots, i_{p} \in I_{\mathcal{C}}$ and $\lambda_{p^{\prime}+1}, \ldots, \lambda_{p}>0$, we have that $R_{\mathcal{C}} \subseteq R_{z^{\prime}, b^{\prime}}$, hence $u_{z^{\prime}, \mathcal{C}} \leq b^{\prime}$.

Given $i_{1}, \ldots, i_{p} \in I \backslash I_{\mathcal{C}}$, if $K_{2}=K_{3}=\emptyset$ and $\lambda_{1} b_{i_{1}}-\lambda_{2} b_{i_{2}}-\ldots-\lambda_{p} b_{i_{p}}<0 \quad \forall \lambda_{1}, \ldots, \lambda_{p}>0$, it is not necessary to apply Proposition 1 , since $u_{z, \mathcal{C}} \geq 0$ by Corollary 2 and, so, $u_{z, \mathcal{C}}>\lambda_{1} b_{i_{1}}-\lambda_{2} b_{i_{2}}-\ldots-\lambda_{p} b_{i_{p}}$.

If $K_{2}=K_{3}=\emptyset$, without loss of generality we may assume that the family $\mathcal{C}_{0}$ from which $\mathcal{C}$ has been obtained (see above) is formed only by packings $C$ such that $|C|>1$ and $\nexists C^{\prime} \in \mathcal{C}_{0} \backslash\{C\}$ with $C^{+} \subseteq C^{\prime+}$ and $C^{-} \subseteq C^{\prime-}$ (see Lemma 1). Then by Lemma 4 it follows that, taking $p=1$ in Proposition 1, the redundancy of a constraint induced by a packing will not be detected. 
Lemma 4. Let $r \in I \backslash I_{\mathcal{C}}$ and $z=\sum_{j \in J} a_{r j} x_{j}$. If $r$ is the index of a constraint induced by a packing and $K_{2}=K_{3}=\emptyset$, then $u_{z, \mathcal{C}}>b_{r}$.

Proof. Let $C$ be the packing that induces the $r$ th constraint and let $\mathcal{C}_{0}$ be the family of packings from which $\mathcal{C}$ has been obtained. Since $C \in \mathcal{C}_{0}$, we have that $|C|>1$ and $\nexists C^{\prime} \in \mathcal{C}_{0} \backslash\{C\}$ such that $C^{+} \subseteq C^{\prime+}$ and $C^{-} \subseteq C^{\prime-}$.

If $\left|C^{-}\right|>1$, then $b_{r}<0$, hence $u_{z, \mathcal{C}}>b_{r}$, since $u_{z, \mathcal{C}} \geq 0$.

If $\left|C^{-}\right|=1$, then $\left|C^{+}\right| \geq 1$ and $b_{r}=0$. Thus, considering that $a_{r j}=1 \quad \forall j \in C^{+}$, by Theorem 1 it follows that $u_{z, \mathcal{C}} \geq 1>b_{r}$.

If $\left|C^{-}\right|=0$, then $\left|C^{+}\right| \geq 2, C=C^{+}$and $b_{r}=1$. Therefore, since $r \notin I_{\mathcal{C}}$, it must be $C \nsubseteq C_{k} \quad \forall k \in K$ and, by Theorem 1 , we obtain that $u_{z, \mathcal{C}} \geq 2>b_{r}$.

Example 2 shows a situation where it is possible to detect a redundant constraint by considering it jointly with another constraint, but not by considering it alone.

ExAmple 2. Let (5)-(8) be the constraint system that defines the feasible region of $(P)$.

$$
\begin{aligned}
3 x_{1}+5 x_{2}-x_{3}+x_{4}+6 x_{5} & \leq 10 \\
x_{1}+x_{2}-x_{3}+x_{4}+4 x_{5} & \leq 4 \\
x_{1}+x_{2} & \leq 1 \\
x_{4}+x_{5} & \leq 1
\end{aligned}
$$

Let $\mathcal{C}=\{\{1,2\},\{4,5\}\}$. (Note that constraints (7) and (8) are induced by the packings $\{1,2\}$ and $\{4,5\}$ respectively). By applying Proposition 1 to constraints (5) and (6) we have that $z=\left(3 \lambda_{1}-\lambda_{2}\right) x_{1}+\left(5 \lambda_{1}-\lambda_{2}\right) x_{2}+\left(-\lambda_{1}+\lambda_{2}\right) x_{3}+\left(\lambda_{1}-\lambda_{2}\right) x_{4}+\left(6 \lambda_{1}-4 \lambda_{2}\right) x_{5}$, $u_{z, \mathcal{C}}=\max \left\{5 \lambda_{1}-\lambda_{2}, 0\right\}+\max \left\{\lambda_{1}-\lambda_{2}, 6 \lambda_{1}-4 \lambda_{2}, 0\right\}+\max \left\{-\lambda_{1}+\lambda_{2}, 0\right\}$ and $\lambda_{1} b_{i_{1}}-\lambda_{2} b_{i_{2}}=10 \lambda_{1}-4 \lambda_{2}$.

- If $\lambda_{1} \leq \frac{1}{5} \lambda_{2}$, then $u_{z, \mathcal{C}}=-\lambda_{1}+\lambda_{2}=10 \lambda_{1}-4 \lambda_{2}-11 \lambda_{1}+5 \lambda_{2}>\lambda_{1} b_{i_{1}}-\lambda_{2} b_{i_{2}}$.

- If $\frac{1}{5} \lambda_{2}<\lambda_{1}<\frac{2}{3} \lambda_{2}$, then $u_{z, \mathcal{C}}=5 \lambda_{1}-\lambda_{2}-\lambda_{1}+\lambda_{2}=4 \lambda_{1}=10 \lambda_{1}-4 \lambda_{2}-6 \lambda_{1}+4 \lambda_{2}>$ $\lambda_{1} b_{i_{1}}-\lambda_{2} b_{i_{2}}$.

- If $\frac{2}{3} \lambda_{2} \leq \lambda_{1} \leq \lambda_{2}$, then $u_{z, \mathcal{C}}=5 \lambda_{1}-\lambda_{2}+6 \lambda_{1}-4 \lambda_{2}-\lambda_{1}+\lambda_{2}=10 \lambda_{1}-4 \lambda_{2}=$ $\lambda_{1} b_{i_{1}}-\lambda_{2} b_{i_{2}}$.

- If $\lambda_{1}>\lambda_{2}$, then $u_{z, \mathcal{C}}=5 \lambda_{1}-\lambda_{2}+6 \lambda_{1}-4 \lambda_{2}=11 \lambda_{1}-5 \lambda_{2}=10 \lambda_{1}-4 \lambda_{2}+\lambda_{1}-\lambda_{2}>$ $\lambda_{1} b_{i_{1}}-\lambda_{2} b_{i_{2}}$.

So, to detect the redundancy of constraint (5) it suffices to choose $\lambda_{1}$ and $\lambda_{2}$ such that $\frac{2}{3} \lambda_{2} \leq \lambda_{1} \leq \lambda_{2}$. However, if Proposition 1 is applied only to constraint (5), the redundancy is not detected, since taking $\lambda_{1}=1$ we obtain that $u_{z, \mathcal{C}}=5+6=11>b_{i_{1}}$. 


\section{Conclusions}

In this paper we have presented a new procedure for obtaining upper bounds on linear functions that makes use of the information provided by certain families of packings, coverings and special ordered sets. It can determine better upper bounds than the traditional procedures, which do not consider either coverings or special ordered sets, and it can be particularly useful in problems without packings. We have also presented a new method for detecting redundant constraints in 0-1 linear programming problems based on these upper bounds. It can detect some redundancy situations that the methods available in current literature cannot, since it allows consideration of several constraints jointly, whereas the existing methods consider only single constraints. Consequently, this new method can improve the current preprocessing techniques.

\section{Acknowledgements}

I am deeply grateful for the hospitality shown to me while a visitor at the Department of Artificial Intelligence of the School of Computer Science of the Technical University of Madrid, where I have written this paper.

\section{References}

[1] Atamtürk, A.; Nemhauser, G.L.; Savelsbergh, M.W.P. (2000) "Conflict graphs in solving integer programming problems", European Journal of Operational Research 121(1): 40-55.

[2] Bixby, R.E.; Ceria, S.; McZeal, C.M.; Savelsbergh, M.W.P. (1998) "An updated mixed integer programming library: MIPLIB 3.0", Technical Report TR98-03, Department of Computational and Applied Mathematics, Rice University. Available from URL:

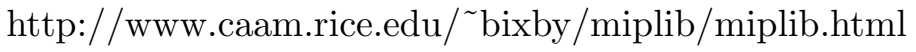

[3] Crowder, H.; Johnson, E.L.; Padberg, M. (1983) "Solving large-scale zero-one linear programming problems", Operations Research 31(5): 803-834.

[4] Dietrich, B.L.; Escudero, L.F.; Garín, A.; Pérez, G. (1993) "O(n) procedures for identifying maximal cliques and non-dominated extensions of consecutive minimal covers and alternates", Top 1(1): 139-160.

[5] Escudero, L.F.; Garín, A.; Pérez, G. (1996) "On using clique overlapping for detecting knapsack constraint redundancy and infeasibility in 0-1 mixed integer programs", Top 4(1): 87-98.

[6] Escudero, L.F.; Muñoz, S. (1998) "On characterizing tighter formulations for 0-1 programs", European Journal of Operational Research 106(1): 172-176.

[7] Guignard, M.; Spielberg, K. (1981) "Logical reduction methods in zero-one programming. Minimal preferred variables", Operations Research 29(1): 49-74. 
[8] Hoffman, K.L.; Padberg, M. (1991) "Improving LP-representations of zero-one linear programs for branch-and-cut", ORSA Journal on Computing 3(2): 121-134.

[9] Johnson, E.L.; Kostreva, M.M.; Suhl, U.H. (1985) "Solving 0-1 integer programming problems arising from large scale planning models", Operations Research 33(4): 803-819.

[10] Johnson, E.L.; Nemhauser, G.L.; Savelsbergh, M.W.P. (2000) "Progress in linear programming-based algorithms for integer programming: An exposition", INFORMS Journal on Computing 12(1): 2-23.

[11] Muñoz, S. (1995) "A correction of the justification of the Dietrich-Escudero-GarínPérez $O(n)$ procedures for identifying maximal cliques and non-dominated extensions of consecutive minimal covers and alternates", Top 3(1): 161-165.

[12] Muñoz, S. (1999) Reforzamiento de Modelos en Programación Lineal 0-1. Tesis Doctoral, Universidad Complutense de Madrid, Madrid.

[13] Nemhauser, G.L.; Wolsey, L.A. (1988) Integer and Combinatorial Optimization. John Wiley, New York.

[14] Savelsbergh, M.W.P. (1994) "Preprocessing and probing techniques for mixed integer programming problems", ORSA Journal on Computing 6(4): 445-454. 